American Medical Journal 2 (2): 111-118, 2011

ISSN 1949-0070

(C) 2011 Science Publications

\title{
Lifestyles and Migraine Attack
}

\author{
${ }^{1}$ Hui Jun Chih and ${ }^{2}$ Wenbin Liang \\ ${ }^{1}$ Curtin Health Innovation Research Institute, School of Public Health, \\ ${ }^{2}$ National Drug Research Institute, Faculty of Health Science, \\ Curtin University, GPO Box U1987, Perth, Western Australia 6845, Australia
}

\begin{abstract}
Problem statement: Migraine is a headache disorder of serious public health concerns as many sufferers are permanently disabled and incapable of carrying out simple daily routines. Migraine is contributed by biological and lifestyle factors. Understanding the association between these factors and migraine, particularly the modifiable lifestyle factors, can reduce the prevalence of this chronic disease and ease the burden on the health care system. Approach: Unilabiate and multivariate logistic regression methods were used to analyse the prevalence of migraine among the participants who completed the Australian National Health Survey during the 2007-2008 period. Demographical and lifestyle factors including age, gender, Body Mass Index (BMI), social marital status, equivalent household income, remoteness of residence, daily fruits and vegetables intake, participation in physical activities, alcohol use, tobacco smoking and distress level were investigated in this study. Results: The prevalence of migraine is on average 1.27 times higher among those who aged between 35-44 years old $(95 \% \mathrm{CI}=1.03,1.57)$ compared to the other age groups, 2.24 times higher among females $(95 \%$ $\mathrm{CI}=1.89,2.67)$ compared to the males and 2.70 times higher among those who reported high to very high stress level $(95 \% \mathrm{CI}=2.23,3.27)$ compared to those who reported low to medium stress level. The prevalence of migraine of those participating in the recommended level of physical activity is 0.69 times of those who did not meet the recommended level $(95 \% \mathrm{CI}=0.57,0.84)$. The prevalence of migraine among the medium risk drinker and high risk drinker is 0.68 times $(95 \% \mathrm{CI}=0.49,0.92)$ and 0.42 times $(95 \% \mathrm{CI}=0.27,0.68)$, respectively, of the low risk drinker. Conclusion: The findings of this study suggested that engaging in physical activity and reduce the level of stress may reduce the risk of migraine attack.
\end{abstract}

Key words: Body Mass Index (BMI), Australian Standard Geographical Classification (ASGC), National Health and Medical Research Council (NHMRC), Total Metabolic Equivalent (MET), serious headache disorder

\section{INTRODUCTION}

Migraine, a serious headache disorder, is a severe public health issue as it is affecting the workforce and family with many sufferers classified as being disabled for work and even daily activities (WHO, 2006). The prevalence of migraine varied across the continents and is the lowest for Asian and African men and women (2.0-2.5\% for men and 6.9-7.7\% for women), higher for European (5.7\% of European men and $14.7 \%$ of European women) and the highest among South/Central American and North American (6.9-7.3\% for men and $16.3-21.5 \%$ for women) (Lipton and Bigal, 2005). On average, about $6 \%$ of men and $14 \%$ of women around the world are affected by current migraine (Stovner $e t$ al., 2007). In addition, it is associated with other comorbidities, such as stroke and other cardiovascular diseases, respiratory diseases and allergies and depression (Schur et al., 2009; Diener et al., 2008). The World Health Organization (WHO) has urged the development of preventive measures for migraine (WHO, 2006). However, effective programs rely on good identification of the key precipitating factors of migraine. The difference in the prevalence across the continents suggests the likelihood of genetic make-ups and lifestyle factors as the key contributors to migraine.

Corresponding Author: Hui Jun Chih, School of Public Health, Curtin Health Innovation Research Institute, Curtin University, GPO Box U1987, Perth, Western Australia 6845, Australia 
This has prompted research into this area to further confirm this assumption.

Studies have been carried out to look at the risk factors of migraine. Data from twin and family studies showed familial aggregation of migraine, suggesting that genetic factors may play important roles in the development of migraine (Schur et al., 2009; Nyholt et al., 2009; Ulrich et al., 1999; Gardner, 2006; Montagna, 2000). There is also some evidence about the effect of environmental factors such as seasonal change of temperature, sunlight and noise on migraine attacks (Mulder et al., 2003; Friedman and Dye, 2009; Hansen et al., 2011; Chakravarty et al., 2009 Zivadinov et al., 2003; Queiroz et al., 2009). Meanwhile, studies done in the United States of America, Finland, Denmark, Sweden, Spain, Croatia, Brazil, Japan and Hong Kong suggested that socioeconomic factors such as education level, income level, types of occupation and marital status as well as lifestyle factors including alcohol drinking, tobacco smoking, physical inactivity and stress level are also key influential factors of migraine (Lipton and Bigal, 2005; Le et al., 2011; Maki et al., 2008; Molarius et al., 2008; Fernandez-de-las-Penas et al., 2010; Panconesi, 2008; Vo et al., 2011; Cheung, 2000). However, there remain controversial statements about the association of these lifestyle factors in increasing or lowering the prevalence of migraine, in particular the association between migraine, physical activity, smoking and drinking amongst Brazilian, Danish, Spanish and Swedish population (Queiroz et al., 2009; Le et al., 2011; Molarius et al., 2008; Fernandez-delas-Penas et al., 2010). One possible explanation of the ' $\mathrm{J}$ ' or 'inverted J' shape relationship between these factors and migraine, as reported by some of the studies, is that the migraine sufferers recognized those factors as the precipitating factors of migraine and have modified their lifestyles to cope with it, the 'sick quitter' phenomenon (Shaper et al., 1988).

Despite the amount of cross-sectional studies conducted around the world on the relationship between lifestyle factors and migraine, the current literature lacks information on the Australian. Nevertheless, 59\% of Australians drink, 19\% smoke and $65 \%$ engage in some moderate to vigorous physical activity (ABS, 2008) with $45 \%$ of Australian suffering from life-long mental distress (ABS, 2008). The interaction of these factors on the prevalence of migraine warrants further investigation to understand the relationship between migraine and lifestyle factors in Australia. The aim of the study is to explore the association between lifestyle factors, health characteristics, social status and living environments (drinking, smoking, physical activity, daily fruits and vegetables intake, BMI, stress level, income level, marital status and remoteness of living area) and the prevalence of migraine in Australia.

\section{MATERIALS AND METHODS}

This is a population-based cross-sectional study using the data collected during the 2007-2008 Australian National Health Survey (NHS) by the Australian Bureau of Statistics (ABS). Participants were 18 years or older at the time of interviews. There were 9929 subjects who provided answers to all variables included in the multivariate regression analysis and these data are included in this study. The study collected detailed information on the health status of the population, use of health services and other actions people had recently taken for their health, health-related aspects of lifestyle (including daily fruits and vegetables intake, participation in physical activity), risky behaviour (including frequency and quantity of alcohol use, tobacco smoking and distress level), as well as demographic characteristics (age, gender, Body Mass Index (BMI), social marital status, equivalent household income, remoteness of residence). Information was obtained through face-to-face interviews by trained ABS interviewers. Details of the survey sampling strategy and data collection methods are described elsewhere (ABS, 2009a). A copy of the questionnaire can be obtained from the ABS website: www.abs.gov.au

Demographical data were used in the analysis as potential predictors of migraine. Gender, age, social marital status and equivalent household income were categorized following the ABS method while the remoteness of residence was grouped according to the Australian Standard Geographical Classification (ASGC) and Body Mass Index (BMI) was grouped as underweight, normal weight, overweight and obese for $<18.5, \quad 18.5-24.9, \quad 25-29.9, \quad>30 \quad \mathrm{~kg} \mathrm{~m} \mathrm{~m}^{-2}$, respectively. Lifestyle factors, including daily intake of fruits and vegetables, physical activity, alcohol drinking behaviour, tobacco smoking and distress level were also used to predict the prevalence of migraine (one for with migraine, two for without migraine). Similarly, the daily intake of fruits and 
vegetables were grouped into two groups according to the National Health and Medical Research Council (NHMRC) Dietary Guidelines for Australian adults: (1) lower than the recommended five serves of vegetables and two serves of fruits per day for adults (NHMRC, 2003), (2) on or above the recommended daily intake. The current physical activity guidelines for Australian adults of "at least 30 min of moderate intensity physical activity on most days" is adopted in this study (DOHA, 2005). Specifically, the physical activity level is defined according to the Australian NHS's method, where the level of physical activity is calculated and expressed in terms of Total Metabolic Equivalent (MET) score by multiplying the number of times activity undertaken in last week by the average time per session (minutes) by the intensity (3.5 for walking, 5.0 for moderate exercise or 7.5 for vigorous exercise). A total MET score of 800 or higher for the last week before the Australian NHS was defined as meeting the current physical activity guidelines for Australian adults (ABS, 2009b). Alcohol drinking behaviour was categorized into five categories with those who have never consumed alcohol as abstainer, those who had ever consumed alcohol for the past 12 months as ever drinker and those consumed alcohol in the last week were further categorized into three groups based on the 2000 National guideline (NHMRC, 2001): $<50 \mathrm{~mL}, 50-75 \mathrm{~mL},>75 \mathrm{~mL}$ of pure alcohol as low risk drinkers, medium risk drinkers and high risk drinkers, respectively, among men; $<25 \mathrm{~mL}, 25$ $50 \mathrm{~mL},>50 \mathrm{~mL}$ of pure alcohol as low risk drinkers, medium risk drinkers and high risk drinkers, respectively, among women. Tobacco smoking status was categorized into four groups: currently smoke daily; currently smoke weekly (less frequent than daily); ex-smoker (did not smoke at the time of the Australian NHS, but had smoked more than 100 cigarettes in the past); and never smoker. Stress level was measured by Kessler psychological distress scale and were grouped into low-moderate (10-21) group and high-very high (22-50) group.

Univariate and multivariate logistic regression models were used to investigate the associations between presence of migraine (dependent variable) and lifestyles (independent variables). In the multivariate analysis, age, BMI, daily intake of fruits and vegetables, physical activity level, Kessler psychological distress scale, equivalent household income, remoteness of residence, social marital status, alcohol drinking behaviour and tobacco smoking status were all included in the genderstratified model. The significance level was set at 0.05 for these analyses. Logistic regression model was used to analyse the association. Odds ratio is an appropriate estimate of prevalence when prevalence of the outcome of interest (such as migraine) is low (i.e., lower than $15 \%$ in the population) (Vandenbroucke et al., 2007; Walter, 2000; Greenland, 1987). Analysis was performed with STATA 11.

\section{RESULTS}

The demographic and lifestyle factors used to predict the self-reported migraine attacks are presented in Table 1. Both the Univariate and Multivariate analyses show that the prevalence of migraine is higher among those aged between 35-44 years old and lower among those aged 60 years old and above $(\mathrm{p}<0.05)$, after controlling for the other variables. The present study found that females have higher prevalence of migraine compared to the males $(p<0.05)$. The prevalence of migraine is also higher among those who reported a high to very high stress level, compared to those who self-reported a low to moderate stress level $(\mathrm{p}<0.05)$. As illustrated by the Univariate analysis, readers should also note that the prevalence of migraine is higher $(\mathrm{p}<0.05)$ among those who are obese Table 1.

On the other hand, the prevalence of migraine is lower among those who follow a healthy lifestyle Table 1. In particular, the prevalence of migraine is lower among those who engaged in physical activity at the recommended level. Interestingly, the prevalence of migraine is lower among the medium risk drinker and high risk drinker when compared to the other drinker types. After controlling for the other variables, there are also evidences suggesting that those who earn a higher income, consume the recommended daily intake of fruits and live in the remote area of Australia have a lower prevalence of migraine Table 1. Similar trend of migraine prevalence was observed when the data was analysed separately for males and females Table 2. It is evident from this study that age, physical activity, stress level and alcohol drinking behaviour are significantly associated with migraine $(\mathrm{p}<0.05)$. 
Am. Med. J. 2 (2): 111-118, 2011

Table 1: Odds Ratio (OR) of demographical and lifestyle factors for migraine attacks

\begin{tabular}{|c|c|c|c|c|c|c|c|c|c|}
\hline \multirow[b]{2}{*}{ Gender } & & \multicolumn{4}{|c|}{ Univariate } & \multicolumn{4}{|c|}{ Multivariate } \\
\hline & & \multicolumn{3}{|l|}{ OR } & \multirow[t]{2}{*}{$95 \% \mathrm{CI}$} & \multicolumn{3}{|l|}{ OR } & \multirow[t]{2}{*}{$95 \% \mathrm{CI}$} \\
\hline & Males (Reference) & & & & & (Refe & rence) & & \\
\hline & Females & 2.38 & 2.02 & 2.81 & $\mathrm{p}<0.001$ & 2.24 & 1.89 & 2.67 & $\mathrm{p}<0.001$ \\
\hline \multirow[t]{5}{*}{ Age (years old) } & 18-34 (Reference) & & & & & \multicolumn{3}{|c|}{ (Reference) } & \\
\hline & $35-44$ & 1.31 & 1.07 & 1.6 & $P=0.009$ & 1.27 & 1.03 & 1.57 & $\mathrm{P}=0.024$ \\
\hline & $45-59$ & 0.98 & 0.80 & 1.2 & N.S. & 0.92 & 0.74 & 1.14 & N.S. \\
\hline & $60-79$ & 0.5 & 0.39 & 0.64 & $\mathrm{p}<0.001$ & 0.47 & 0.36 & 0.62 & $\mathrm{p}<0.001$ \\
\hline & 80 and above & 0.21 & 0.11 & 0.41 & $\mathrm{p}<0.001$ & 0.19 & 0.1 & 0.39 & $\mathrm{p}<0.001$ \\
\hline \multirow[t]{5}{*}{ BMI } & & & & & & & & & \\
\hline & Normal & & & & & \multicolumn{3}{|c|}{ (Reference) } & \\
\hline & Underweight & 0.83 & 0.45 & 1.52 & N.S. & 0.73 & 0.4 & 1.35 & N.S. \\
\hline & Overweight & 1.06 & 0.90 & 1.26 & N.S. & 1.02 & 0.86 & 1.21 & N.S. \\
\hline & Obese & 1.68 & 1.14 & 2.48 & $\mathrm{P}=0.009$ & 1.42 & 0.96 & 2.12 & N.S. \\
\hline \multirow[t]{3}{*}{ Daily intake of fruits } & & & & & & & & & \\
\hline & $<2$ serves (Reference) & & & & & \multicolumn{2}{|c|}{ (Reference) } & & \\
\hline & $\geq 2$ serves & 0.85 & 0.73 & 1 & $\mathrm{P}=0.047$ & 0.91 & 0.77 & 1.07 & N.S. \\
\hline \multirow[t]{3}{*}{ Daily intake of vegetables } & & & & & & & & & \\
\hline & $<5$ serves (Reference) & & & & & \multicolumn{2}{|c|}{ (Reference) } & & \\
\hline & $\geq 5$ serves & 0.89 & 0.68 & 1.16 & N.S. & 0.95 & 0.72 & 1.24 & N.S. \\
\hline \multirow[t]{3}{*}{ Physical activity } & & & & & & & & & \\
\hline & $<$ Recommended level (F & & & & & (Refe & rence) & & \\
\hline & $\geq$ Recommended level & 0.64 & 0.53 & 0.77 & $\mathrm{p}<0.001$ & 0.69 & 0.57 & 0.84 & $\mathrm{p}<0.001$ \\
\hline \multicolumn{10}{|c|}{ Kessler psychological distress scale } \\
\hline & \multicolumn{4}{|c|}{ Low/moderate level (Reference) } & & \multicolumn{2}{|c|}{ (Reference) } & & \\
\hline \multirow{4}{*}{ Equivalent household income } & High/very high level & 2.82 & 2.36 & 3.38 & $\mathrm{p}<0.001$ & 2.7 & 2.23 & 3.27 & $\mathrm{p}<0.001$ \\
\hline & Low (Reference) & & & & & \multicolumn{2}{|c|}{ (Reference) } & & \\
\hline & Medium & 0.73 & 0.59 & 0.91 & $\mathrm{P}=0.005$ & 0.83 & 0.66 & 1.04 & N.S. \\
\hline & High & 0.66 & 0.53 & 0.82 & $\mathrm{p}<0.001$ & 0.86 & 0.67 & 1.1 & N.S. \\
\hline Remoteness of residence & & & & & & & & & \\
\hline & Major cities of Australia & & & & & (Refe & rence) & & \\
\hline & Inner regional Australia & 1.14 & 0.94 & 1.37 & N.S. & 1.12 & 0.92 & 1.36 & N.S. \\
\hline & Other remote areas & 0.77 & 0.59 & 1 & N.S. & 0.75 & 0.57 & 0.99 & $\mathrm{P}=0.041$ \\
\hline Social marital status & & & & & & & & & \\
\hline & Married ${ }^{\#}$ (Reference) & & & & & (Refe & rence) & & \\
\hline & Not married & 1.05 & 0.9 & 1.23 & N.S. & 0.97 & 0.82 & 1.14 & N.S. \\
\hline Alcohol drinking behaviour & & & & & & & & & \\
\hline & Low risk drinker (Refere & & & & & (Refe & rence) & & \\
\hline & Medium risk drinker & 0.71 & 0.52 & 0.96 & $\mathrm{P}=0.026$ & 0.68 & 0.49 & 0.92 & $P=0.014$ \\
\hline & High risk drinker & 0.49 & 0.31 & 0.78 & $\mathrm{P}=0.003$ & 0.42 & 0.27 & 0.68 & $\mathrm{p}<0.001$ \\
\hline & Ever drinker & 1.1 & 0.92 & 1.3 & N.S. & 1 & 0.83 & 1.19 & N.S. \\
\hline & Abstainer & 0.96 & 0.72 & 1.29 & N.S. & 0.89 & 0.65 & 1.21 & N.S. \\
\hline Tobacco smoking status & & & & & & & & & \\
\hline & Currently smoke daily (R & & & & & (Refe & rence) & & \\
\hline & Currently smoke weekly & 0.91 & 0.51 & 1.61 & N.S. & 1 & 0.55 & 1.79 & N.S. \\
\hline & Ex-smoker & 1.03 & 0.83 & 1.28 & N.S. & 1.22 & 0.97 & 1.53 & N.S. \\
\hline & Never smoker & 0.83 & 0.68 & 1.01 & N.S. & 0.98 & 0.79 & 1.22 & N.S. \\
\hline
\end{tabular}

${ }^{\#}$ :including de facto relationship; N.S. $=$ p >0.05

Table 2: Gender-stratified odds ratio for migraine attacks

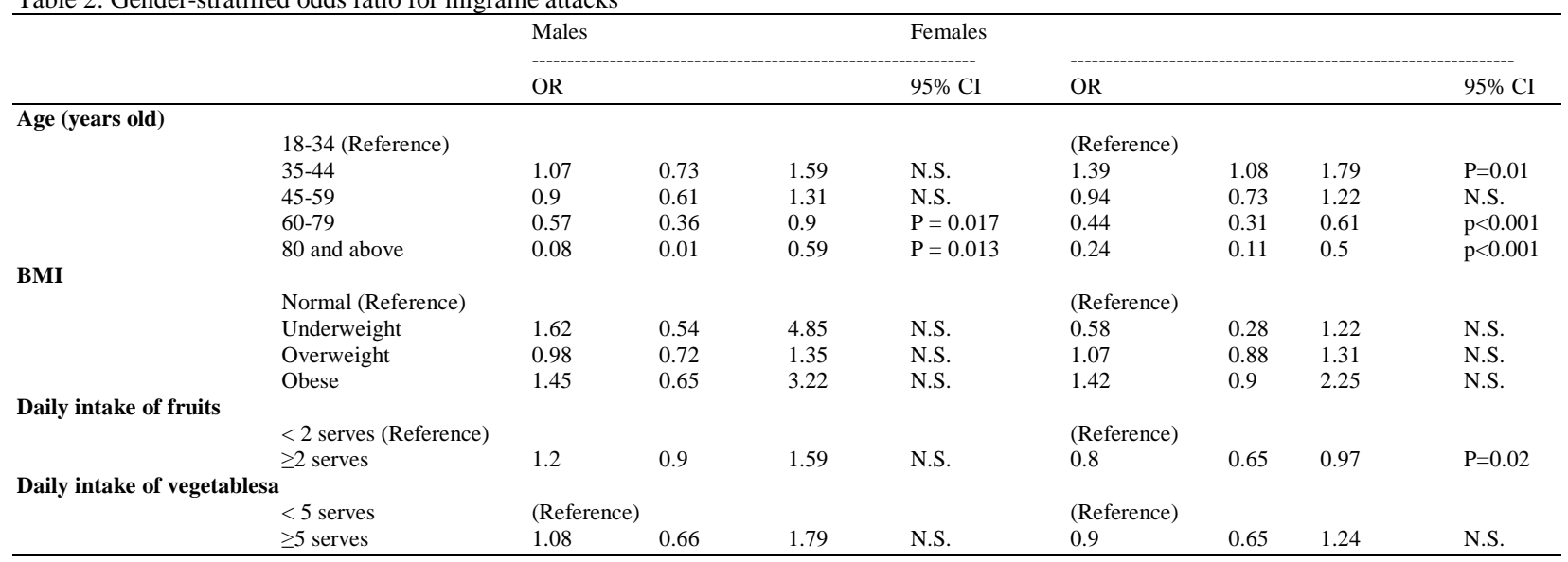


Am. Med. J. 2 (2): 111-118, 2011

\begin{tabular}{|c|c|c|c|c|c|c|c|c|c|}
\hline \multicolumn{10}{|l|}{ Physical activity } \\
\hline & \multicolumn{5}{|c|}{$<$ Recommended level (Reference) } & \multicolumn{4}{|l|}{ (Reference) } \\
\hline & \multirow{2}{*}{\multicolumn{9}{|c|}{ Kessler psychological distress scale }} \\
\hline & & & & & & & & & \\
\hline & $\mathrm{High} / \mathrm{very}$ high level & 3.64 & 2.6 & 5.08 & $\mathrm{p}<0.001$ & 2.38 & 1.89 & 3.01 & $\mathrm{p}<0.001$ \\
\hline \multicolumn{10}{|c|}{ Equivalent household income } \\
\hline & \multicolumn{5}{|l|}{ Low (Reference) } & \multicolumn{4}{|l|}{ (Reference) } \\
\hline & Medium & 1.00 & 0.66 & 1.52 & N.S. & 0.75 & 0.57 & 0.98 & $P=0.035$ \\
\hline & High & 0.95 & 0.61 & 1.48 & N.S. & 0.81 & 0.6 & 1.09 & N.S. \\
\hline \multicolumn{10}{|c|}{ Remoteness of residence } \\
\hline & \multicolumn{5}{|c|}{ Major cities of Australia (Reference) } & \multicolumn{4}{|l|}{ (Reference) } \\
\hline & Inner regional Australia & 1.27 & 0.9 & 1.77 & N.S. & 1.04 & 0.82 & 1.32 & N.S. \\
\hline & Other remote areas & 0.72 & 0.44 & 1.17 & N.S. & 0.76 & 0.55 & 1.06 & N.S. \\
\hline \multicolumn{10}{|c|}{ Social marital status } \\
\hline & \multicolumn{5}{|l|}{ Married \# (Reference) } & \multicolumn{4}{|l|}{ (Reference) } \\
\hline & Not married & 1.14 & 0.85 & 1.52 & N.S. & 0.91 & 0.75 & 1.11 & N.S. \\
\hline & \multicolumn{9}{|c|}{ Alcohol drinking behaviour } \\
\hline & Low risk drinker & \multicolumn{4}{|c|}{ (Reference) } & \multicolumn{4}{|l|}{ (Reference) } \\
\hline & Medium risk drinker & 0.83 & 0.47 & 1.45 & N.S. & 0.6 & 0.41 & 0.88 & $P=0.008$ \\
\hline & High risk drinker & 0.42 & 0.21 & 0.85 & $P=0.015$ & 0.42 & 0.22 & 0.8 & $P=0.008$ \\
\hline & Ever drinker & 1.19 & 0.86 & 1.66 & N.S. & 0.9 & 0.73 & 1.12 & N.S. \\
\hline & Abstainer & 1.24 & 0.66 & 2.36 & N.S. & 0.78 & 0.55 & 1.11 & N.S. \\
\hline \multirow{2}{*}{\multicolumn{10}{|c|}{ Tobacco smoking status }} \\
\hline & \multicolumn{5}{|c|}{ Currently smoke daily (Reference) } & \multicolumn{4}{|l|}{ (Reference) } \\
\hline & Currently smoke weekly & 0.57 & 0.17 & 1.9 & N.S. & 1.25 & 0.63 & 2.49 & N.S. \\
\hline & Ex-smoker & 1.13 & 0.77 & 1.67 & N.S. & 1.26 & 0.95 & 1.67 & N.S. \\
\hline & Never smoker & 0.95 & 0.65 & 1.39 & N.S. & 1.01 & 0.78 & 1.32 & N.S. \\
\hline
\end{tabular}

${ }^{\#}$ : including de facto relationship; N.S. = p >0.05

\section{DISCUSSION}

The present study investigated the associations between selected social and lifestyle factors and prevalence of self-reported migraine attacks among Australians with the 2007-2008 Australian NHS data. The results presented in Table 1 showed that those being women, aged between 35-44 years old and experiencing high to very high stress level reported a higher prevalence of migraine than the other groups. The finding agrees with many of the previous studies conducted across the continents (Queiroz et al., 2009; Molarius et al., 2008; Fernandez-De-Las-Penas et al., 2010; Bigal and Lipton, 2006; Wong et al., 1995; Yokoyama et al., 2009; Aamodt et al., 2006).

Female sex hormones are known as the precipitating factors of migraine (MacGregor et al., 2011; Gupta et al., 2007; Martin and Lipton, 2008). This corresponds to our finding which shows that the prevalence of migraine is higher among women when compared to the males. The underlying cause for association between stress and migraine is not entirely clear; however it is certain that similar to many other chronic diseases (Liang and Lee, 2011), serious form of migraine increases the stress level among patients. There is also some data suggesting that stress may trigger migraine attack or increase the degree of headache (Wober et al., 2007; Leistad et al., 2006; Wacogne et al., 2003). Given the two-way association between stress and migraine, it is important to help migraine patients to develop stress coping skills (Silberstein, 2000; Deeromram et al., 2010). The present study also observed that the prevalence of migraine is lower among those age over 60 years old, those who live outside the major cities and regional areas and among high income earners (Table 1). The relationship between age and hormonal change is plausible to explain the 'protective' effect of ageing against migraine among females (Nyholt et al., 2009). The finding from the present study suggested that those who live outside the major cities lead a more relaxing lifestyle and experience low to moderate level of stress, which corresponds to the finding from the present study that higher stress level can increase the prevalence of migraine. Another plausible explanation is that the areas outside major cities and areas of Australia may not be exposed to high noise level (Ismail et al., 2010), which is another precipitating factor of migraine (Friedman and Dye, 2009; Martin et al., 2006).

The inverse relationship between exercise and the prevalence of migraine observed from the present study remain consistent with the findings of previous studies (Queiroz et al., 2009; Le et al., 2011; Molarius et al., 2008; Varkey et al., 2008). Physical activities reduce stress level (Norris et al., 1992; Fox, 1999) and may further reduce risk of migraine attack. Studies have also established a hypothesis that exercise is effective in regulating the aetiology of migraine by releasing hormones and nitric oxide in the cerebrovascular 
system (Queiroz et al., 2009; Yokoyama et al., 2009; Lockett and Campbell, 1992). However, further experimental trials are necessary to investigate the validity of these assumptions. It is also interesting to note that the medium and high risk drinkers reported a lower prevalence of migraine. This inverted "J" shape finding is consistent with the previous findings from Japan, Spain, Norway and Austria (Fernandez-de-lasPenas et al., 2010; Yokoyama et al., 2009; Aamodt et al., 2006; Wober et al., 2007). The scenario is believed to be attributable to the sick-quitter behaviour (Shaper et al., 1988) as health professionals advises migraine sufferers to avoid the precipitating factor, such as alcohol (Panconesi, 2008; Kelman, 2007).

This study is a population-based study with relative large and representative sample of the Australian population. The main limitation of the present study is that the nature of a cross-sectional study does not allow the investigation into the actual causal relationship of these potential risk factors and migraine. Future prospective studies are necessary to confirm the relationship.

\section{CONCLUSION}

The findings of this study suggested that engaging in physical activity and reduce the level of stress may reduce the risk of migraine attack.

\section{ACKNOWLEDGEMENT}

The reaches declare no conflict of interest but would like to express our gratitude to the Australian Bureau of Statistics for providing the data collected from the 2007-2008 Australian NHS.

\section{REFERENCES}

Aamodt, A.H., L.J. Stovner, K. Hagen, G. Brathen and J. Zwart, 2006. Headache prevalence related to smoking and alcohol use. The Head-HUNT Study. Eur. J. Neurol., 13: 1233-1238. PMID: 17038038

ABS, 2008. National Survey of Mental Health and Wellbeing: Summary of Results, 2007. Canberra: ABS.

ABS, 2009a. National Health Survey: Summary of Results, 2007-2008. ABS, Canberra.

ABS, 2009b. National Health Survey: users' guide electronic publication. Australian Bureau of Statistics, Canberra.

Bigal, M.E. and R.B. Lipton, 2006. Modifiable risk factors for migraine progression. Headache: $\mathrm{J}$. Head Face Pain, 46: 1334-1343. DOI: 10.1111/j.1526-4610.2006.00577.x
Chakravarty, A., A. Mukherjee and D. Roy, 2009. Trigger factors in childhood migraine: A clinicbased study from Eastern India. J. Headache Pain, 10: 375-380. PMID: 19705059

Cheung, R.T., 2000. Prevalence of migraine, tensiontype headache and other headaches in Hong Kong. Headache: J. Head Face Pain, 40: 473-479. PMID: 10849044

Deeromram, C., A. Suwannimitr and S. Jundeekrayom, 2010. Mental health promotion among nursing students. J. Soc. Sci., 6: 133-140. DOI: 10.3844/jssp.2010.133.140

Diener, H.C., M. Kuper and T. Kurth, 2008. Migraineassociated risks and comorbidity. J. Neurol., 255: 1290-1301. PMID: 18958572

DOHA, 2005. National physical activity guidelines for adults. Department of Health and Ageing, Canberra.

Fernandez-de-las-Penas, C., V. Hernandez-Barrera, P. Carrasco-Garrido, C. Alonso-Blanco and D. Palacios-Cena et al., 2010. Population-based study of migraine in Spanish adults: Relation to sociodemographic factors, lifestyle and co-morbidity with other conditions. J. Headache Pain, 11: 97104. PMID: 20012124

Fox, K.R., 1999. The influence of physical activity on mental well-being. Public Health Nutr., 2: 411418. PMID: 10610081

Friedman, D.I. and T.D.V. Dye, 2009. Migraine and the environment. Headache: J. Head Face Pain, 49: 941-952. PMID: 19545255

Gardner, K.L., 2006. Genetics of migraine: An update. Headache: J. Head Face Pain, 46: S19-S24. DOI: 10.1111/j.1526-4610.2006.00486.x

Greenland, S., 1987. Interpretation and choice of effect measures in epidemiologic analyses. Am. J. Epidemiol., 125: 761-768. PMID: 3551588

Gupta, S., S. Mehrotra, C.M. Villalon, M. Perusquia and P.R. Saxena et al., 2007. Potential role of female sex hormones in the pathophysiology of migraine. Pharmacol. Ther., 113: 321-340. PMID: 17069890

Hansen, J.M., A.W. Hauge, M. Ashina and J. Olesen, 2011. Trigger factors for familial hemiplegic migraine. Cephalalgia, 31: 1274-1281. PMID: 21784774

Ismail, A.R., M.H.M. Haniff, C.B. Kim, B.M. Deros and N.K. Makhtar, 2010. A survey on environmental factors and job satisfaction among operators in automotive industry. Am. J. Applied Sci., 7: 556-561. 
Kelman, L., 2007. The triggers or precipitants of the acute migraine attack. Cephalalgia, 27: 394-402. PMID: 17403039

Le, H., P. Tfelt-Hansen, A. Skytthe, K. Kyvik and J. Olesen, 2011. Association between migraine, lifestyle and socioeconomic factors: A populationbased cross-sectional study. J. Headache Pain, 12: 157-172. PMID: 21390550

Leistad, R.B., T. Sand, R.H. Westgaard, K.B. Nilsen and L.J. Stovner, 2006. Stress-induced pain and muscle activity in patients with migraine and tension-type headache. Cephalalgia, 26: 64-73. PMID: 16396668

Liang, W. and A.H. Lee, 2011. Does childhood asthma increase the risk of mood disorders in later life? Am. Med. J., 2: 93-97. DOI: 10.3844/amjsp.2011.93.97

Lipton, R.B. and M.E. Bigal, 2005. Migraine: Epidemiology, impact and risk factors for progression. Headache: J. Head Face Pain, 45: S3S13. DOI: 10.1111/j.1526-4610.2005.4501001.x

Lockett, D.M. and J.F. Campbell, 1992. The effects of aerobic exercise on migraine. Headache: J. Head Face Pain, 32: 50-54. DOI: 10.1111/j.15264610.1992.hed3201050.x

MacGregor, E.A., J.D. Rosenberg and T. Kurth, 2011. Sex-related differences in epidemiological and clinic-based headache studies. Headache: J. Head Face Pain, 51: 843-859. DOI: 10.1111/j.15264610.2011.01904.x

Maki, K., J. Vahtera, M. Virtanen, M. Elovainio and L. Keltikangas-Jarvinen et al., 2008. Work stress and new-onset migraine in a female employee population. Cephalalgia, 28: 18-25. DOI: 10.1111/j.1468-2982.2007.01462.x

Martin, P.R., J. Reece and M. Forsyth, 2006. Noise as a trigger for headaches: Relationship between exposure and sensitivity. Headache: J. Head Face Pain, 46: 962-972. DOI: 10.1111/j.15264610.2006.00468.x

Martin, V.T. and R.B. Lipton, 2008. Epidemiology and biology of menstrual migraine. Headache: J. Head Face Pain, 48: S124-S130. DOI: 10.1111/j.15264610.2008.01310.x

Molarius, A., А. Tegelberg and J. Ohrvik, 2008. Socioeconomic factors, lifestyle and headache disordersa population-based study in sweden. Headache: J. Head Face Pain, 48: 1426-1437. DOI: 10.1111/j.1526-4610.2008.01178.x

Montagna, P., 2000. Molecular genetics of migraine headaches: a review. Cephalalgia, 20: 3-14. DOI: 10.1046/j.1468-2982.2000.00003.x
Mulder, E.J., C. van Baal, D. Gaist, M. Kallela, J. Kaprio, D.A. Svensson et al., 2003. Genetic and environmental influences on migraine: A twin study across six countries. Twin Res., 6: 422-431. PMID: 14624726

NHMRC, 2001. Australian Alcohol Guidelines: Health Risks and Benefits. 1st Edn., Australian National Health and Medical Research Council, Canberra, ISBN: 1864961546, pp: 128.

NHMRC, 2003. Food for Health-Dietary Guidelines for Australian Adults. 1st Edn., NAtional Health and Medical Research Council, Canberra, ISBN: 1864961414, pp: 472.

Norris, R., D. Carroll and R. Cochrane, 1992. The effects of physical activity and exercise training on psychological stress and well-being in an adolescent population. J. Psychosom. Res., 36: 5565. PMID: 1538350

Nyholt, D.R., N.G. Gillespie, K.R. Merikangas, S.A. Treloar and N.G. Martin et al., 2009. Common genetic influences underlie comorbidity of migraine and endometriosis. Genetic Epidemiol., 33: 105-113. PMID: 18636479

Panconesi, A., 2008. Alcohol and migraine: trigger factor, consumption, mechanisms. A review. J. Headache Pain, 9: 19-27. PMID: 18231712

Queiroz, L.P., M.F. Peres, E.J. Piovesan, F. Kowacs and M.C. Ciciarelli et al., 2009. A nationwide population-based study of migraine in Brazil. Cephalalgia, 29: 642-649. PMID: 19187337

Schur, E.A., C. Noonan, D. Buchwald, J. Goldberg and N. Afari, 2009. A twin study of depression and migraine: Evidence for a shared genetic vulnerability. Headache: J. Head Face Pain, 49: 1493-1502. DOI: 10.1111/j.15264610.2009.01425.x

Shaper, A.G., G. Wannamethee and M. Walker, 1988. Alcohol and mortality in british men: Explaining the u-shaped curve. Lancet, 2: 1267-1273. PMID: 2904004

Silberstein, S.D., 2000. Practice parameter: Evidencebased guidelines for migraine headache (an evidence-based review): Report of the quality standards subcommittee of the american academy of neurology. Neurology, 55: 754-762. PMID: 10993991

Stovner, L.J., K. Hagen, R. Jensen, Z. Katsarava and R. Lipton et al., 2007. The global burden of headache: a documentation of headache prevalence and disability worldwide. Cephalalgia, 27: 193-210. PMID: 17381554 
Ulrich, V., M. Gervil, K.O. Kyvik, J. Olesen and M.B. Russell, 1999. Evidence of a genetic factor in migraine with aura: A population-based Danish twin study. Ann. Neurol., 45: 242-246. PMID: 9989627

Vandenbroucke, J.P., E.V. Elm, D.G. Altman, P.C. Gotzsche and C.D. Mulrow et al., 2007. Strengthening the Reporting of Observational Studies in Epidemiology (STROBE): Explanation and elaboration. Ann. Int. Med., 147: 167-164. PMID: 17938389

Varkey, E., K. Hagen, J.A. Zwart and M. Linde, 2008. Physical activity and headache: Results from the Nord-Trøndelag Health Study (HUNT). Cephalalgia, 28: 1292-1297. PMID: 18771495

Vo, M., A. Ainalem, C. Qiu, B.L. Peterlin and S.K. Aurora et al., 2011. Body mass index and adult weight gain among reproductive age women with migraine. Headache: J. Head Face Pain, 51: 559569. DOI: 10.1111/j.1526-4610.2010.01833.x

Wacogne, C., J.P. Lacoste, E. Guillibert, F.C. Hugues and C.L. Jeunne, 2003. Stress, anxiety, depression and migraine. Cephalalgia, 23: 451-455. PMID: 12807524

Walter, S.D., 2000. Choice of effect measure for epidemiological data. J. Clin. Epidemiol., 53: 931939. PMID: 11004419
WHO, 2006. Neurological Disorders: Public Health Challenges. 1st Edn., World Health Organization, Geneva, ISBN: 9241563362, pp: 218.

Wober, C., W. Brannath, K. Schmidt, M. Kapitan and E. Rudel et al., 2007. Prospective analysis of factors related to migraine attacks: The PAMINA study. Cephalalgia, 27: 304-314. PMID: 17376107

Wong, T.W., K.S. Wong, T.S. Yu and R. Kay, 1995. Prevalence of migraine and other headaches in Hong Kong. Neuroepidemiology, 14: 82-91. PMID: 7891818

Yokoyama, M., T. Yokoyama, K. Funazu, T. Yamashita and S. Kondo et al., 2009. Associations between headache and stress, alcohol drinking, exercise, sleep, and comorbid health conditions in a Japanese population. J. Headache Pain, 10: 177185. PMID: 19326184

Zivadinov, R., K. Willheim, D. Sepic-Grahovac, A. Jurjevic and M. Bucuk et al., 2003. Migraine and tension-type headache in Croatia: A populationbased survey of precipitating factors. Cephalalgia, 23: 336-343. 